\title{
The DOTI Approach: An Alternative Way of Teaching and Learning the Chain Rule
}

\author{
Steve Anglin \\ Department of Mathematics, Case Western Reserve University, Cleveland, OH, USA
}

How to cite this paper: Anglin, A. (2018). The DOTI Approach: An Alternative Way of Teaching and Learning the Chain Rule. The Educational Review, USA, 2(11), 555-558 http://dx.doi.org/10.26855/er.2018.11.005

Corresponding author: Steve Anglin, Sc.M., Ph.D.(h.c.), Department of Mathematics, Case Western Reserve University, Cleveland, OH, USA.

\begin{abstract}
For composite functions $\mathrm{f}(\mathrm{x})$ and $\mathrm{g}(\mathrm{x})$, the derivative is the chain rule, a chain of derivatives on $\mathrm{f}$, then $\mathrm{g}$. Well, a new or alternative way of teaching, learning and thinking of the Chain Rule is as follows: Similar to learning how to multiply/factor two binomials using the FOIL Method, we hereby propose the DOTI (Differentiate "Outside" To "Inside") approach as a way of best teaching or learning the Chain Rule for taking the derivative of two or more composite functions, $f$ and $g$, etc.
\end{abstract}

\section{Keywords}

DOTI Approach, Learning, the Chain Rule, Derivative, Differentiate, Calculus

\section{Introduction}

For composite functions $\mathrm{f}(\mathrm{x})$ and $\mathrm{g}(\mathrm{x})$, the derivative is the chain rule, a chain of derivatives on $\mathrm{f}$, then $\mathrm{g}$. Well, a new or alternative way of teaching, learning and thinking of the Chain Rule could be as follows:

Similar to learning how to multiply/factor two binomials using the FOIL Method, we propose the DOTI acronym which means Differentiate "Outside" To "Inside" approach as an alternative way of best teaching or learning the Chain Rule for taking the derivative of two or more composite functions, $f$ and $g$, etc.

Why DOTI? DOTI is just an alternative approach to teaching and learning the Chain Rule. Undergraduate students seem to more often gravitate towards acronyms or other easy way of remembering a method of calculation. This is a proposed approach that could be taught and learned more broadly. See (Anglin, 2012) below for a talk given on DOTI at the Joint Mathematics Meetings held by MAA and AMS.

\section{Definition}

Differentiation occurs first on the "outside" on the function $\mathrm{f}(\mathrm{x})$, then "inside" on the second function, $\mathrm{g}(\mathrm{x})$.

$\bigvee(f \backslash \operatorname{circ} g)^{\prime}=\left(f^{\prime} \backslash \operatorname{circ} g\right)^{*} g \backslash$

$(f \circ g)^{\prime}=\left(f^{\prime} \circ g\right) * g^{\prime}$

$f=f(x), g=g(x)$

The above definition is found in most Calculus text books. 


\section{Examples}

\subsection{Example 1}

$$
\begin{aligned}
& (f \circ g)=5\left(x^{4}-x^{2}+x\right)^{4} \\
& f=5 u^{4}, g=x^{4}-x^{2}+x=u \\
& (f \circ g)^{\prime}=\left(f^{\prime} \circ g\right) * g^{\prime} \\
& f^{\prime}=20 u^{3}, g^{\prime}=4 x^{3}-2 x+1=u \\
& \therefore(f \circ g)^{\prime}=20\left(x^{4}-x^{2}+x\right)^{3} *\left(4 x^{3}-2 x+1\right)
\end{aligned}
$$

See (Wolfram Alpha LLC., 2018) below under references for confirmation.

Again, differentiation occurs first on the "outside" on the function $\mathrm{f}(\mathrm{x})$, then "inside" on the second function, $\mathrm{g}(\mathrm{x})$.

\subsection{Example 2}

$$
\begin{aligned}
& f=3 x^{3}, g=x^{2}-4=u \\
& (f \circ g)=3 x^{3}\left(x^{2}-4\right) \\
& f^{\prime}=9 x^{2}, g^{\prime}=2 x=u \\
& (f \circ g)^{\prime}=\left(f^{\prime} \circ g\right) * g^{\prime} \\
& \therefore(f \circ g)^{\prime}=9 x^{2}\left(x^{2}-4\right) * 2 x \\
& \quad(f \circ g)^{\prime}=18 x^{3}\left(x^{2}-4\right)
\end{aligned}
$$

See (Wolfram Alpha LLC., 2018) below under references for confirmation.

Differentiation occurs first on the "outside" on the function $\mathrm{f}(\mathrm{x})$, then "inside" on the second function, $\mathrm{g}(\mathrm{x})$. However, in this case, it's a little harder to see, but it can work.

\subsection{Example 3}

$$
\begin{aligned}
& f=3 e^{u}, g=x^{2}=u \\
& (f \circ g)=3 \exp \left(x^{2}\right) \\
& f^{\prime}=3 e^{u}, g^{\prime}=2 x=u \\
& (f \circ g)^{\prime}=\left(f^{\prime} \circ g\right) * g^{\prime} \\
& \therefore(f \circ g)^{\prime}=3 \exp \left(x^{2}\right) * 2 x
\end{aligned}
$$




$$
(f \circ g)^{\prime}=6 x e^{x^{2}}
$$

See [5.] under References for more information/confirmation.

And here, below, you can differentiate on the "outside" on the function $\mathrm{f}(\mathrm{x})$, then "inside" on the second function, $\mathrm{g}(\mathrm{x})$.

\subsection{Example 4}

$$
\begin{aligned}
& f=3 \cos ^{4}(u), g=x^{2}=u \\
& (f \circ g)=3 \cos ^{4}\left(x^{2}\right) \\
& f^{\prime}=-12 \cos ^{3}(u) \sin (u), g^{\prime}=2 x \\
& (f \circ g)^{\prime}=\left(f^{\prime} \circ g\right) * g^{\prime} \\
& \therefore(f \circ g)^{\prime}=\left(-12 \cos ^{3}\left(x^{2}\right) \sin \left(x^{2}\right)\right) * 2 x \\
& \quad(f \circ g)^{\prime}=-24 x \cos ^{3}\left(x^{2}\right) \sin \left(x^{2}\right)
\end{aligned}
$$

See (Wolfram Alpha LLC., 2018) for further details or confirmation of the above example.

And lastly, below, you can differentiate on the "outside" on the function $\mathrm{f}(\mathrm{x})$, then "inside" on the second function, $\mathrm{g}(\mathrm{x})$, and further inside on the third function introduced below as $\mathrm{h}(\mathrm{x})$.

\subsection{Example 5}

$$
\begin{aligned}
& f=u^{3}, g_{1}=3 x^{-2}, g_{2}=v^{5}, u=g_{1}+g_{2}, v=5 x^{3}-7 x=h \\
& \left.\left(f \circ\left(g_{1}+g_{2}\right) \circ h\right)=\left[3 x^{-2}+\left(5 x^{3}-7 x\right)^{5}\right)\right]^{3} \\
& f^{\prime}=3 u^{2}, g_{1}^{\prime}=-6 x^{-3}, g_{2}^{\prime}=5 v^{4}, \\
& u^{\prime}=g_{1}^{\prime}+g_{2}^{\prime}=-6 x^{-3}+5 v^{4}, v^{\prime}=15 x^{2}-7=h^{\prime} \\
& \left(f \circ\left(g_{1}+g_{2}\right) \circ h\right)^{\prime}=f^{\prime}\left(g_{1}+g_{2}(h)\right) *\left[g_{1}^{\prime}+g_{2}^{\prime}(h)\right] * h^{\prime} \\
& \left.\therefore\left(f \circ\left(g_{1}+g_{2}\right) \circ h\right)^{\prime}=3\left[3 x^{-2}+\left(5 x^{3}-7 x\right)^{5}\right)\right]^{2} * \quad\left[-6 x^{-3}+5\left(5 x^{3}-7 x\right)^{4}\right] *\left(15 x^{2}-7\right)
\end{aligned}
$$

See (Socratic Organization, 2018) and (Khan Academy, 2018) on how the above is calculated.

\section{Compliance with Ethical Standards}

The author declares that they have no conflict of interest. 


\section{References}

Anglin. (2012)"Learning the Chain Rule Using the DOTI Method", Joint Mathematics Meetings (AMS.org) presentation / session: http://jointmathematicsmeetings.org/amsmtgs/2141 abstracts/1086-vb-36.pdf .

Wolfram Alpha LLC. 2018. Wolfram|Alpha. http://www.wolframalpha.com/input/?i=derivative+of $+5(\mathrm{x} \% 5 \mathrm{E} 4+-+\mathrm{x} \% 5 \mathrm{E} 2+\% 2 \mathrm{~B}+\mathrm{x}) \% 5 \mathrm{E} 4$ (access October 14, 2018).

Wolfram Alpha LLC. 2018. Wolfram|Alpha. http://www.wolframalpha.com/input/?i=derivative+of+x\%5E2+-+4 (access October 14, 2018).

Wolfram Alpha LLC. 2018. Wolfram|Alpha. http://www.wolframalpha.com/input/?i=derivative+of+3x\%5E3 (access October 14, 2018).

Wolfram Alpha LLC. 2018. Wolfram|Alpha. http://www.wolframalpha.com/input/?i=derivative+of+3exp(x\%5E2) (access October 14, 2018).

Wolfram Alpha LLC. 2018. Wolfram|Alpha. http://www.wolframalpha.com/input/?i=derivative+of+3+cos\%5E4(x\%5E2) (access October 14, 2018).

Socratic Organization. 2018. https://socratic.org/questions/what-is-the-derivative-of-f-g-h-x (access October 14, 2018).

Khan Academy. 2018. http://khanacademy.org (access October 14, 2018). 